\title{
mTOR signaling in osteosarcoma: Oncogenesis and therapeutic aspects (Review)
}

\author{
KAI HU, HAI-BO DAI and ZHI-LONG QIU \\ Department of Orthopedics, Xiangtan Central Hospital, Xiangtan, Hunan 411100, P.R. China
}

Received February 21, 2016; Accepted March 16, 2016

DOI: $10.3892 /$ or.2016.4922

\begin{abstract}
The mammalian target of rapamycin (mTOR) is a serine/threonine protein kinase that belongs to the phosphoinositide-3-kinase (PI3K)-related kinase family. Oncogenic activation of mTOR signaling significantly contributes to the progression of different types of cancers including osteosarcoma (OS; the most common primary malignant tumor of bone). In the present study, we review the association of the mTOR signaling pathway with OS, and the possible effective treatment strategies by targeting this pathway. In the metastatic behavior of $\mathrm{OS}$, one of the most common actionable aberrations was found in the PI3K/Akt/mTOR pathway. Upon phosphorylation, activated mTOR contributes to OS cellular transformation and poor cancer prognosis via downstream effectors such as S6K1, 4EBP1 and eIF4E, which are overexpressed in OS. Targeting the mTOR complex is a significant approach in cancer therapeutic research, and of course, rapamycin is the primary inhibitor of mTOR. Various other chemotherapeutic molecules have also shown potential activity against mTOR. As mTOR is a new promising oncological target and blockade of the mTOR pathway with selective inhibitors has significant potential in OS therapeutic research, the development of the optimal dose, regimen and a rationale for the use of $\mathrm{mTOR}$ inhibitors in combination with other anticancer agents may provide a successful treatment strategy for OS.
\end{abstract}

\section{Contents}

1. Introduction

2. The mTOR complex

3. Association of mTOR signaling with OS

4. Targeting the mTOR complex in osteosarcoma

5. Future direction

Correspondence to: Dr Zhi-Long Qiu, Department of Orthopedics, Xiangtan Central Hospital, Xiangtan, Hunan 411100, P.R. China E-mail: qiuzhilong6049@yahoo.com

Key words: rapamycin, mammalian target of rapamycin, PI3K/Akt/ mTOR pathway, osteosarcoma

\section{Introduction}

Rapamycin, also known as sirolimus is an antifungal agent that contains a macrocyclic lactone. It was isolated from a strain of the soil bacterium, Streptomyces hygroscopicus, found in Easter Island (known locally as Rapa Nui) and named as rapamycin according to the name of its place of discovery $(1,2)$. It was also discovered to possess potent immunosuppressive, antiproliferative and anticancer properties due to its ability to arrest the cell cycle in the G1 phase (3).

The mammalian target of rapamycin (mTOR), also known as FKBP12-rapamycin complex-associated protein (FRAP) is a serine/threonine protein kinase that belongs to the phosphoinositide-3-kinase (PI3K)-related kinase family $(4,5)$. Rapamycin binds with its intracellular receptor FK506-binding protein (FKBP12) and forms a complex (mTOR) and exerts inhibitory activity (6). mTOR is a large, ubiquitously expressed multi-effector protein that in humans is encoded by the MTOR gene. It is composed of 2,549 amino acids with a molecular mass of $\sim 250 \mathrm{kDa}$ (7). The N-terminal half of the protein contains 20 tandem HEAT repeats, each of which consists of $2 \alpha$ helices of $\sim 40$ amino acids, and are implicated in protein-protein interactions. The C-terminal half of mTOR contains the kinase catalytic domain (KIN) that has sequence similarity with the catalytic site in PI3Ks $(6,7)$. It is reported to play a vital role in controlling cell growth, cell survival, angiogenesis and autophagy through the regulation of protein synthesis $(5,8)$.

Deregulation of mTOR activity is associated with numerous types of cancer. Oncogenic activation of mTOR signaling through phosphorylation of PI3K or Akt significantly contributes to the initiation and development of tumors. Over-activation of mTOR activity supports tumor growth by promoting cell cycle progression, increasing cell proliferation, and inhibiting autophagy through its effect on protein synthesis $(9,10)$. Multimodal treatment strategies have been developed to target this pathway resulting in the emergence of unique pharmacological inhibitors that may provide the best therapeutic advantage for the treatment of cancers $(11,12)$.

Osteosarcoma (OS) is the most common primary malignant tumor of bone occurring mostly in children and young adults. It arises from the primitive transformed cells of mesenchymal origin that mainly affects osteoblastic differentiation and produces immature bone $(13,14)$. It mainly occurs in the tubular long bones; however also occurs in other sites such as 
the femur, humerus, knee and pelvis. OS is the eighth most common form of pediatric cancer with a higher incidence in males (5.4/million/year) than in females (4.0/million/year) (15). The typical signs and symptoms of OS include pain followed by the localized swelling and limitations of joint movement (14). The exact etiology of OS is unknown, however it is evident that various risk factors are associated with the development and pathogenesis of OS including age, gender, ethnicity, genetic and familial factors (16). The current treatment options for OS are surgery, chemotherapy and radiotherapy. The standard therapy of OS is a combination of orthopedic surgery along with doxorubicin, cisplatin, adriamycin, high-dose methotrexate with leucovorin rescue and ifosfamide $(14,17)$. Despite the multimodal therapy, the conventional therapy of OS is still unsatisfactory due to the risk of metastasis, recurrence and chemoresistance. Therefore, alternative therapeutic strategies are urgently needed to improve the efficacy and reduce the side-effects. Targeted therapies that can identify targetable aberrations (specific genes or molecular pathways) and can manipulate apoptosis and autophagy have become a promising approach in OS treatment $(18,19)$.

mTOR and Akt signaling have been reported to be involved with the metastatic behavior of OS, and the most common actionable aberrations found in the PI3K/Akt/mTOR pathway $(19,20)$. In the present review, we focused on the association of the mTOR signaling pathway in the pathogenesis of OS, and the possible effective treatment strategies by targeting this pathway.

\section{The mTOR complex}

mTOR is the catalytic subunit of two structurally and functionally distinct multiprotein complexes: mTOR complex 1 (mTORC1) and mTOR complex 2 (mTORC2) (21). mTORC1 is sensitive to the effects of rapamycin and is composed of mTOR itself, regulatory-associated protein of mTOR (Raptor) and the non-core components of $40 \mathrm{kDa}$ pro-rich AKT substrate (PRAS40; also known as AKT1S1) (22). Raptor serves as the binding platform where its association with mTOR forms a nutrient-sensitive complex that signals to regulate cell growth $(4,23)$. Conversely, PRAS40 regulates mTORC1 kinase activity by functioning as a direct inhibitor of mTORC1 substrate binding (24).

mTORC2 is composed of mTOR, rapamycin-insensitive companion of mTOR (RICTOR), mammalian stress-activated map kinase-interacting protein 1 (mSIN1; also known as MAPKAP1) and protein observed with RICTOR (PROTOR). RICTOR is known as a scaffold protein that contributes to the structural foundation of mTORC2, and plays an essential role in embryonic growth and development. However, the functions of mSIN1 and PROTOR are not clear $(8,25)$. Mammalian lethal with SEC13 protein 8 [mLST8; also known as G protein $\beta$ subunit-like (G $\beta \mathrm{L})$ ] and DEP domaincontaining mTOR-interacting protein (DEPTOR) are the two other proteins common in both complexes (26). The function of mLST8 within mTORC1 is not clear. However, it is essential for the activation of mTORC2 that regulates growth and development. DEPTOR functions as an inhibitor of mTOR signaling that inhibits mTORC1 and mTORC2 activity by preventing substrate binding to mTORC1 and mTORC2 $(27,28)$.
The activity of mTORC1 is stimulated by growth factors, insulin, phosphatidic and amino acid (particularly leucine) levels, energy status and oxidative stress $(4,29)$. Insulin receptor substrate (IRS) activates PI3K through stimulation of growth factors. PI3K generates phosphatidylinositol 3,4,5-triphosphate (PIP3) upon phosphorylation. PIP3 then promotes the phosphorylation of protein kinase (PKB/AKT) by 3-phosphoinositide-dependent protein kinase-1 (PDK1). AKT phosphorylates tuberous sclerosis complex (TSC) which ultimately leads to the activation of mTORC1 $(22,30)$. Activated mTORC1 phosphorylates its two downstream effectors, p70 ribosomal S6 kinase 1 (p70S6K1 or S6K1) and the eukaryotic initiation factor 4E (eIF4E) binding protein 1 (4E-BP1), which are thought to be the major regulators of protein translation, cell proliferation, angiogenesis and autophagy. Autophagy is an evolutionarily conserved, self-degradation system of cellular components through an autophagosomal-lysosomal pathway dependent on nutrient conditions and is reported to be deregulated in cancer. During starvation, mTORC1 is inactivated and dissociates from the ULK1 kinase complex (a protein complex composed of Atg1, Atg13, Atg17 and Atg101) to induce autophagy $(6,31,32)$.

Akt is the major substrate of mTORC2 and the full activation of Akt requires two steps of phosphorylation: firstly, PDK1-mediated phosphorylation at a threonine T308 residue and secondly, mTORC2-mediated phosphorylation at a serine S473 residue. The mTORC2 is activated by growth factors and functions as an important regulator for the organization of the actin cytoskeleton, cell survival and lipid metabolism $(8,33)$.

\section{Association of mTOR signaling with OS}

mTOR is a critical signaling pathway that is potentially activated in OS. However, the mechanism by which the mTOR signaling pathway is activated in OS is poorly understood. Evidence shows that multiple elements of mTOR signaling are involved in OS (Fig. 1). The upstream regulators (for example, PI3K) of mTOR signaling are deregulated through the overexpression of growth factor receptors, such as human epidermal growth factor receptor 2 (HER-2) and insulin-like growth factor receptor (IGFR), mutations in $P I 3 K$ and amplifications of $A K T$ (34-36). PTEN, the negative regulator of PI3K signaling is downregulated in OS and contributes to bone proliferation, angiogenesis and metastasis through several mechanisms, including mutation, methylation or protein instability $(34,37)$. The downstream effectors of mTOR (such as, S6K1, 4EBP1 and eIF4E) are overexpressed in OS and implicated in cellular transformation and poor cancer prognosis (38).

Ezrin, a member of the ezrin/radixin/moesin (ERM) family of proteins, is involved in intracellular signal transduction (39). It is reported that ezrin expression increases phosphorylation and expression of S6K1 and 4E-BP-1 as well as S6K1 activity, which may induce ezrin-mediated metastatic behavior in OS (40). Zhou et al investigated the expression of mTOR and p70S6K in 65 patients with primary OS, and found that overexpression both of mTOR and p70S6K are significantly correlated with OS cell proliferation, survival and metastasis in OS patients (38). Tenascin-C (TN-C) is one of extracellular matrix glycoproteins found to be overexpressed in OS. Tanaka et al reported that the overexpression of TN-C 


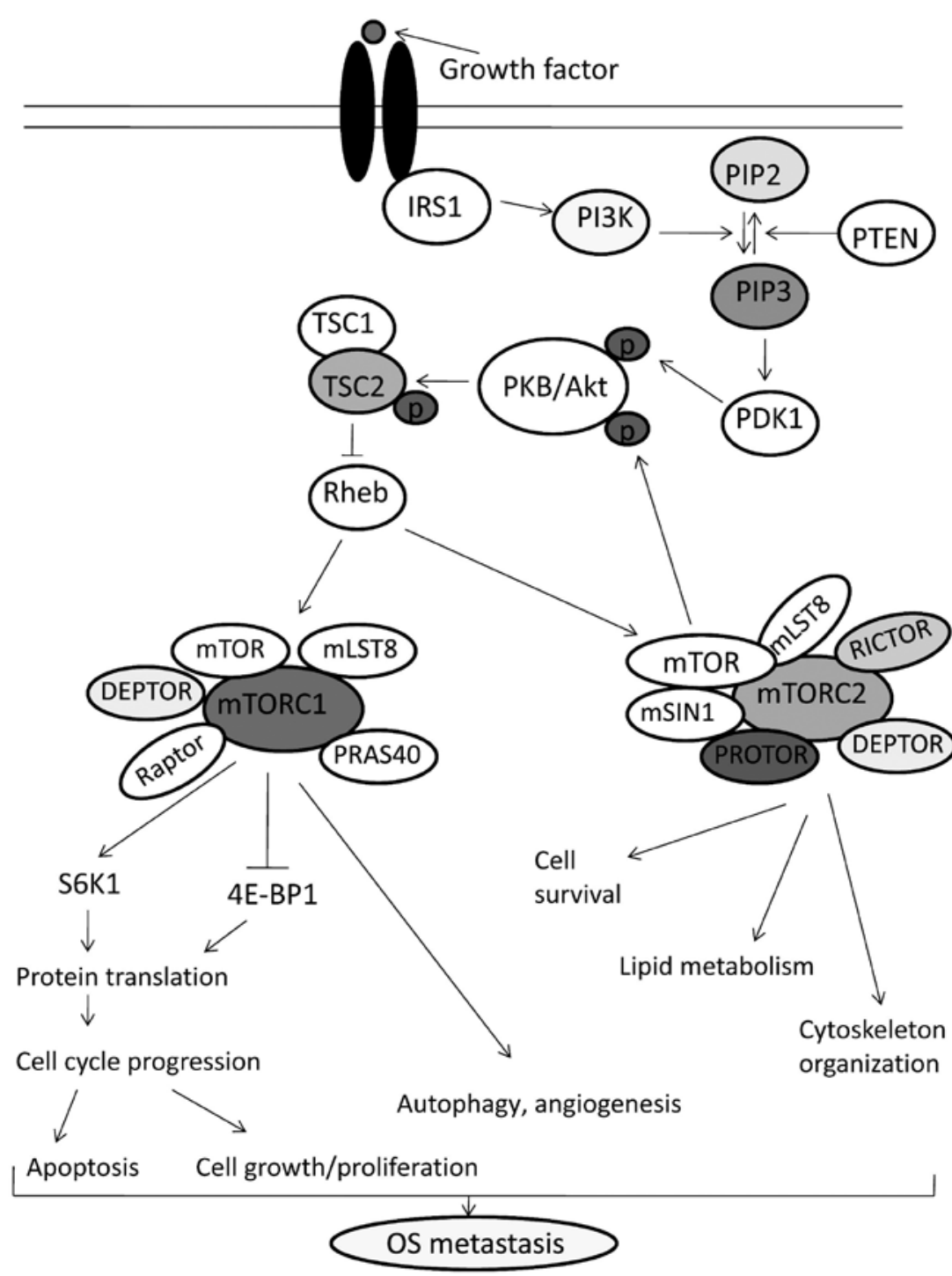

Figure 1. mTOR signaling pathway and its association with osteosarcoma. Upon growth factor stimulation, insulin receptor substrate (IRS) activates PI3K and PI3K generates PIP3, which in turn activates PDK1 and Akt. After phosphorylation by PDK1, Akt phosphorylates and inhibits the TSC complex which ultimately leads to the activation of mTORC1 $(22,30)$. Activated mTORC1 phosphorylates S6K1 and 4E-BP1, which thereby regulate protein translation, cell proliferation, angiogenesis and autophagy $(4,32)$. mTORC2 activates Akt through the two steps of phosphorylation and functions as an important regulator for the organization of the actin cytoskeleton, cell survival and lipid metabolism $(8,33)$. The oncogenic activation of the components of the mTOR pathway through the overexpression of growth factor receptors, mutations in PI3K, loss of PTEN and amplifications of AKT contributes to bone proliferation, angiogenesis, autophagy and inhibits apoptosis leading to OS metastasis (34).

has an effect of easier migration of OS cells to promote distant metastases (41). Zheng et al reported that TN-C in combination with its alternative spliced FNIII repeats with A1 subdomain (TN-C FNIIIA1) is overexpressed in MG-63 OS cells and promote cellular migration. Their study also found that the downstream molecules of mTOR such as, 4E-BP1 and S6K1 can facilitate the expression of TN-C FNIIIA1 in MG-63 cells and promote the metastasis of OS by facilitating MG-63 cell migration (42).

\section{Targeting the mTOR complex in osteosarcoma}

Conventional therapy for OS remains unsatisfactory due to the resistance of OS to chemotherapy and radiotherapy. New alternative therapies and therapeutic targets are needed to be identified for the development of more effective target-specific
anti-OS agents for the treatment of OS. Targeting the mTOR signaling pathway may represent an attractive potential therapeutic approach for the prevention and treatment of OS $(43,44)$. Table I summarizes the potential therapeutic strategies and their effects on OS by targeting the mTOR signaling pathway.

Perry et al used several methods to identify genomic events contributing to OS, and suggested that targeting the $\mathrm{PI} 3 \mathrm{~K} / \mathrm{mTOR}$ pathway has central vulnerability for therapeutic exploitation in OS (34). Rapamycin itself is the inhibitor of the mTOR signaling pathway, and may be a promising agent against OS. Zhao et al evaluated the effects of rapamycin on human OS cells and reported that rapamycin increased the expression of p27 and decreased the expression of cyclin D1, inhibited OS cell proliferation, induced autophagy and cell cycle arrest in the G1 phase. Their results also reported that rapamycin suppressed the tumor growth in mouse xenograft 
Table I. Potential therapeutic strategies of OS by targeting the mTOR pathway.

\begin{tabular}{|c|c|c|c|}
\hline Therapeutic agents & Experimental OS samples & Effects & (Refs.) \\
\hline Rapamycin & Murine model of OS (K7M2) & Inhibited ezrin-related metastatic behavior & $(40)$ \\
\hline Rapamycin & Human OS cells & $\begin{array}{l}\text { Inhibited OS cell proliferation, suppressed } \\
\text { tumor growth, induced autophagy and } \\
\text { cell cycle arrest in the G1 phase }\end{array}$ & $(43)$ \\
\hline Rapamycin or CDDP & MG63 OS cells & $\begin{array}{l}\text { Enhanced the effects of the activation of } \\
\text { autophagy and induced apoptosis }\end{array}$ & $(50)$ \\
\hline $\begin{array}{l}\text { Kinase inhibitor (PP242) } \\
\text { or rictor knockdown } \\
\text { by siRNA }\end{array}$ & $\begin{array}{l}\text { OS cell lines } \\
\text { (MG63/U2OS/SaOS-2) }\end{array}$ & Prevented cell migration and induced apoptosis & $(47)$ \\
\hline NVP-BEZ235 & $\begin{array}{l}\text { OS cell lines } \\
(\mathrm{MG} 63, \mathrm{U} 2 \mathrm{OS} \text { and SaOS-2) }\end{array}$ & $\begin{array}{l}\text { Induced apoptosis and cell cycle arrest, } \\
\text { slowed tumor progression and } \\
\text { reduced tumor vasculature }\end{array}$ & $(52,53)$ \\
\hline Alisertib & U2OS and MG63 OS cells & Promoted autophagy and induced apoptosis & $(54)$ \\
\hline $\begin{array}{l}\text { Sorafenib plus } \\
\text { everolimus }\end{array}$ & $\begin{array}{l}\text { OS cell lines (MNNG-HOS, HOS, } \\
\text { KHOS/NP, MG63, U2OS, SJSA-1 } \\
\text { and SaOS-2), patients with } \\
\text { unresectable high-grade OS }\end{array}$ & $\begin{array}{l}\text { Enhanced antiproliferative and proapoptotic } \\
\text { effects impaired tumor growth, potentiated } \\
\text { antiangiogenesis, reduced migratory } \\
\text { and metastatic potential }\end{array}$ & $(20,56)$ \\
\hline Ridaforolimus & Patients with OS & Achieved confirmed partial response & $(57)$ \\
\hline Lupeol & MNNG-HOS and MG63 OS cells & $\begin{array}{l}\text { Decreased tumor growth, induced apoptosis } \\
\text { and cell cycle arrest }\end{array}$ & $(58)$ \\
\hline FIM-A & Human OS cells & $\begin{array}{l}\text { Inhibited cell growth and proliferation } \\
\text { and reduced angiogenesis }\end{array}$ & $(59)$ \\
\hline Oleanolic acid & MG63 and SaOS-2 cells & Exhibited antitumor activity & $(60)$ \\
\hline $\begin{array}{l}\text { Geldanamycin plus } \\
\text { 3-methyladenine }\end{array}$ & KTHOS cells & Induced autophagy and apoptosis & $(18)$ \\
\hline MicroRNA-223 & MG63 cells & $\begin{array}{l}\text { Inhibited cell growth, exhibited significant } \\
\text { G0/G1 arrest and increased apoptosis }\end{array}$ & $(61)$ \\
\hline MicroRNA-101 & OS tissues and $\mathrm{SaOS}-2$ cells & Inhibited cell proliferation and promoted apoptosis & $(62)$ \\
\hline Perifosine & Human OS cells & Induced cell apoptosis and growth inhibition & $(63)$ \\
\hline RAD001 plus zoledronate & Human and mouse OS cells & $\begin{array}{l}\text { Inhibited cell proliferation and } \\
\text { abolished drug resistance }\end{array}$ & $(64)$ \\
\hline
\end{tabular}

OS, osteosarcoma; mTOR, mammalian target of rapamycin.

models (43). The use of mTOR inhibitors blunted the p53 response to nucleolar stress by regulating ribosome biogenesis and mRNA translation (45). p53 potently inhibited cell proliferation, metastasis, and angiogenesis in OS cells through the inhibition of the PI3K/AKT/mTOR pathway (46). Wang et al reported that targeting of $\mathrm{mTORC} 2$ either by a kinase inhibitor or rictor knockdown prevented OS cell migration and promoted cisplatin-induced apoptosis (47).

Although mTOR is a novel promising oncological target, unfortunately mTOR-targeted monotherapies have shown only modest antitumor activity in OS. The combination with other rationally selected therapeutic agents may improve the response (48). Wagner et al evaluated the activity of the combined inhibition of cixutumumab [inhibitor of insulin-growth factor type 1 receptor (IGF-1R)] with the mTOR inhibitor temsirolimus in preclinical models of OS and other sarcoma patients. In the present study, 43 evaluable patients received $6 \mathrm{mg} / \mathrm{kg}$ cixutumumab with $8 \mathrm{mg} / \mathrm{m}^{2}$ temsirolimus intravenously once weekly in 4-week cycles and observed that $16 \%$ of patients were progression-free at 12 weeks. The reported adverse effects were only mucositis, electrolyte disturbances and myelosuppression (49). In another study, Xie et al reported that rapamycin enhanced the effects of the activation of autophagy and the induction of apoptosis of OS cells by cis-diamminedichloroplatinum (cisplatin) (50). In another study, Wan et al found that blockade of the mTOR pathway with rapamycin inhibited ezrin-related metastatic behavior in a murine model of OS (40). In a recent study, the combination of rapamycin and an autophagy inhibitor, specific and potent autophagy inhibitor-1 (spautin-1) was found to effectively induce the apoptosis pathway, and was suggested as a possible treatment option in OS (51). 
Systematic screening has identified that dual inhibition of the PI3K-mTOR pathway is a sensitive, druggable target in OS (52). Zhu et al studied the therapeutic potential of NVP-BEZ235, a novel PI3K/mTOR dual inhibitor, on OS cells, and found that NVP-BEZ235 downregulated cyclin D1/B1 expression, induced apoptosis and cell cycle arrest in the G0/ G1 phase through inhibition of PI3K-AKT-mTORC1 signaling in OS cells. An in vivo study also showed that oral administration of NVP-BEZ235 inhibited OS xenograft growth in SCID mice (53). In a murine pre-clinical model, Gobin et al reported that NVP-BEZ235 significantly reduced tumor progression, ectopic tumor bone formation and reduced tumor vasculature (54). Alisertib (ALS; MLN8237) is a selective aurora kinase-A inhibitor that displays potent growth inhibitory, pro-apoptotic, pro-autophagic and EMT inhibitory effects on OS cells. Niu et al reported that ALS markedly downregulated the expression levels of cyclin D1/2 and B1, induced G2/M arrest, suppressed EMT-like phenotypes as well as promoted apoptosis and autophagy via inhibition of the PI3K/Akt/mTOR signaling pathway in OS cells (55). MLN0128, an ATP-competitive mTOR kinase inhibitor was reported to inhibit mTORC1/2 targets and show potent antitumor activity in multiple sarcoma subtypes including OS (56).

Treatment with the multikinase inhibitor sorafenib showed disease stabilization and reduced drug resistance in patients with unresectable advanced and metastatic OS (20,57). Pignochino et al investigated the activity of sorafenib in combination with everolimus in preclinical models of OS to overcome the drawbacks. Their results observed enhanced antiproliferative and proapoptotic effects, impaired tumor growth, potentiated antiangiogenesis as well as reduced migratory and metastatic potential by complete inhibition of the mTOR pathway (20). A phase II clinical trial (ClinicalTrials.gov, no. NCT01804374) was conducted, in which 38 patients with unresectable high-grade OS were enrolled. Patients received $800 \mathrm{mg}$ sorafenib plus $5 \mathrm{mg}$ everolimus once a day until disease progression and observed progression-free survival at 6 months in 17 patients. The most common clinical adverse events were lymphopenia and hypophosphataemia, oral mucositis, diarrhoea, hand and foot syndrome, thrombocytopenia, fatigue and anaemia. No treatment-related death was reported in the present study (57). Another phase II trial was conducted to assess the antitumor activity of ridaforolimus (an inhibitor of mTOR) in patients with distinct subtypes of advanced bone and soft tissue sarcomas. In this trial, a total of 212 patients were enrolled and ridaforolimus $(12.5 \mathrm{mg})$ was administered as a 30 -min intravenous infusion once daily for 5 days every 2 weeks. Two patients with OS achieved confirmed partial response. Related adverse events were stomatitis, mouth ulceration, mucosal inflammation and fatigue (58).

Liu et al investigated the anticancer activity of lupeol (a dietary triterpene present in many fruits and medicinal plants) in human OS cells. Their results showed that lupeol promoted downregulation of the protein expression levels of PI3K, AKT, p70S6K and cyclin D1 and upregulation of the expression levels of p21 and p27, which play a pivotal role in the regulation of apoptosis and cell cycle arrest in G0/G1 phase of OS cells in vitro. They also established tumor xenografts in female nude BALB/c mice, and administered lupeol intravenously and found that administration of lupeol decreased tumor growth, induced apoptosis as well as cell cycle arrest of human OS cells through the PI3K/AKT/mTOR signaling pathway (59). Phosphorus-containing sirolimus (FIM-A) inhibited cancer cell growth and proliferation, reduced angiogenesis, induced cell cycle arrest in the G1 phase of OS cells by targeting mTOR. In in vivo mouse osteosarcoma xenografts, FIM-A significantly decreased phosphorylation of p70S6K1 and 4E-BP1, and decreased the average tumor volume and the number of intratumoral microvessels through the imhibition of mTORC1 signaling (60). Zhou et al examined the anticancer activities of oleanolic acid (OA; a pentacyclic triterpenoid) in OS cells, and found that $\mathrm{OA}$ inhibited cell proliferation, induced $\mathrm{G} 1$ arrest in OS cells and regulated protein translation through the inhibition of mTOR signaling (61).

Heat shock protein 90 (Hsp90) plays a critically important role in tumor cell growth, survival and autophagy. Mori et al examined the effects of the Hsp90 inhibitor, geldanamycin (GA) on OS cells and found that GA induced autophagy and apoptosis in OS cells through inhibition of the Akt/mTOR signaling pathway. They also reported that the combination of GA and the autophagy inhibitor 3-methyladenine (3-MA) may be an effective treatment for OS as this combination effectively suppressed a protective mechanism induced by the Hsp90 inhibitor and enhanced GA-induced apoptosis in OS cells (18). Hsp90B1 plays an oncogenic role and is a target gene of miR-223. Overexpression of miR-223 downregulated Hsp90B1 and showed tumor-suppressor function in OS through the inhibition of the PI3K/Akt/mTOR pathway (62). miR-101 functions as a tumor suppressor, and is reported to be downregulated in OS tissues and cell lines. mTOR gene is a direct target of miR-101 and reintroduction of miR-101 in OS cell line inhibited the proliferation and promoted apoptosis by downregulating mTOR expression (63). Perifosine (Akt inhibitor) is another possible anti-OS agent that induced cell apoptosis and growth inhibition in human OS cells. Perifosine promoted caspase-3, c-Jun N-terminal kinases (JNK) and p53 activation and inhibited survivin expression by disrupting its association with HSP-90 through inhibition of Akt/mTORC1 signaling (64). Moriceau et al investigated the effects of RAD001 (everolimus, a new orally available mTOR inhibitor) on the growth of human and mouse OS cells in combination with zoledronate (ZOL; an anti-osteoporotic drug). They found that the combination of RAD001 with ZOL augmented the inhibition of cell proliferation and abolished the resistance of OS cells to RAD001 through inhibition of PI3K/mTOR signaling (65). A recent interesting finding showed that dual mTORC1/2 inhibition by the potent mTOR kinase inhibitor INK-128 (MLN0128) exerted anti-OS activity in vitro and in vivo (66). However, there is not yet enough evidence. Yet, INK-128 may be further investigated as a novel anti-OS agent.

\section{Future direction}

mTOR is a master regulator of cell growth, proliferation, survival and autophagy through its ability to stimulate mRNA translation, ribosome biogenesis and protein stability. Oncogenic activation of mTOR signaling plays a crucial role in the development of OS. A better understanding of the functions of the mTOR pathway and the molecular mechanism by which it promotes OS may provide the basis to develop a 
potential therapeutic target for the treatment of OS. mTOR is a new promising oncological target and blockade of the mTOR pathway with selective inhibitors represents an attractive and potential target for the treatment of OS. Development of the optimal dose, regimen and a rationale for the use of mTOR inhibitors in combination with other agents may provide a promising therapy for the treatment of OS and could improve the survival rate of OS patients.

\section{References}

1. Vézina C, Kudelski A and Sehgal SN: Rapamycin (AY-22,989), a new antifungal antibiotic. I. Taxonomy of the producing streptomycete and isolation of the active principle. J Antibiot 28: 721-726, 1975.

2. Wullschleger $\mathrm{S}$, Loewith $\mathrm{R}$ and Hall $\mathrm{MN}$ : TOR signaling in growth and metabolism. Cell 124: 471-484, 2006.

3. Benjamin D, Colombi M, Moroni C and Hall MN: Rapamycin passes the torch: A new generation of mTOR inhibitors. Nat Rev Drug Discov 10: 868-880, 2011.

4. Kim DH, Sarbassov DD, Ali SM, King JE, Latek RR, ErdjumentBromage H, Tempst P and Sabatini DM: mTOR interacts with raptor to form a nutrient-sensitive complex that signals to the cell growth machinery. Cell 110: 163-175, 2002.

5. Laplante $M$ and Sabatini DM: mTOR signaling in growth control and disease. Cell 149: 274-293, 2012.

6. Hay N and Sonenberg N: Upstream and downstream of mTOR. Genes Dev 18: 1926-1945, 2004

7. Hoeffer CA and Klann E: mTOR signaling: At the crossroads of plasticity, memory and disease. Trends Neurosci 33: 67-75, 2010.

8. Zoncu R, Efeyan A and Sabatini DM: mTOR: From growth signal integration to cancer, diabetes and ageing. Nat Rev Mol Cell Biol 12: 21-35, 2011.

9. Guertin DA and Sabatini DM: An expanding role for mTOR in cancer. Trends Mol Med 11: 353-361, 2005.

10. $\mathrm{Xu} \mathrm{K}$, Liu $\mathrm{P}$ and Wei $\mathrm{W}$ : mTOR signaling in tumorigenesis. Biochim Biophys Acta 1846: 638-654, 2014.

11. Beauchamp EM and Platanias LC: The evolution of the TOR pathway and its role in cancer. Oncogene 32: 3923-3932, 2013.

12. Fasolo A and Sessa C: Targeting mTOR pathways in human malignancies. Curr Pharm Des 18: 2766-2777, 2012.

13. Luetke A, Meyers PA, Lewis I and Juergens H: Osteosarcoma treatment - where do we stand? A state of the art review. Cancer Treat Rev 40: 523-532, 2014.

14. Bielack S, Carrle D and Casali PG; ESMO Guidelines Working Group: Osteosarcoma: ESMO clinical recommendations for diagnosis, treatment and follow-up. Ann Oncol 20 (Suppl 4): S137-S139, 2009.

15. Ottaviani $\mathrm{G}$ and Jaffe N: The epidemiology of osteosarcoma Cancer Treat Res 152: 3-13, 2009.

16. Ottaviani G and Jaffe N: The etiology of osteosarcoma. Cancer Treat Res 152: 15-32, 2009

17. Anderson PM and Pearson M: Novel therapeutic approaches in pediatric and young adult sarcomas. Curr Oncol Rep 8: 310-315, 2006.

18. Mori M, Hitora T, Nakamura O, Yamagami Y, Horie R, Nishimura $\mathrm{H}$ and Yamamoto T: Hsp90 inhibitor induces autophagy and apoptosis in osteosarcoma cells. Int J Oncol 46: 47-54, 2015.

19. Egas-Bejar D, Anderson PM, Agarwal R, Corrales-Medina F, Devarajan E, Huh WW, Brown RE and Subbiah V: Theranostic profiling for actionable aberrations in advanced high risk osteosarcoma with aggressive biology reveals high molecular diversity: The human fingerprint hypothesis. Oncoscience 1: 167-179, 2014.

20. Pignochino Y, Dell'Aglio C, Basiricò M, Capozzi F, Soster M, Marchiò S, Bruno S, Gammaitoni L, Sangiolo D, Torchiaro E, et al: The combination of sorafenib and everolimus abrogates mTORC1 and mTORC2 upregulation in osteosarcoma preclinical models. Clin Cancer Res 19: 2117-2131, 2013.

21. Betz C and Hall MN: Where is mTOR and what is it doing there? J Cell Biol 203: 563-574, 2013.

22. Ashworth RE and Wu J: Mammalian target of rapamycin inhibition in hepatocellular carcinoma. World J Hepatol 6: 776-782, 2014.
23. Hara K, Maruki Y, Long X, Yoshino K, Oshiro N, Hidayat S, Tokunaga C, Avruch J and Yonezawa K: Raptor, a binding partner of target of rapamycin (TOR), mediates TOR action. Cell 110: 177-189, 2002.

24. Wang L, Harris TE, Roth RA and Lawrence JC Jr: PRAS40 regulates mTORC1 kinase activity by functioning as a direct inhibitor of substrate binding. J Biol Chem 282: 20036-20044, 2007.

25. Mendoza MC,ErEE and Blenis J: The Ras-ERK and PI3K-mTOR pathways: Cross-talk and compensation. Trends Biochem Sci 36: 320-328, 2011.

26. Guertin DA, Stevens DM, Thoreen CC, Burds AA, Kalaany NY, Moffat J, Brown M, Fitzgerald KJ and Sabatini DM: Ablation in mice of the mTORC components raptor, rictor, or mLST8 reveals that mTORC2 is required for signaling to Akt-FOXO and PKCalpha, but not S6K1. Dev Cell 11: 859-871, 2006.

27. Peterson TR, Laplante M, Thoreen CC, Sancak Y, Kang SA, Kuehl WM, Gray NS and Sabatini DM: DEPTOR is an mTOR inhibitor frequently overexpressed in multiple myeloma cells and required for their survival. Cell 137: 873-886, 2009.

28. Laplante M and Sabatini DM: mTOR signaling at a glance. J Cell Sci 122: 3589-3594, 2009.

29. Fang Y, Vilella-Bach M, Bachmann R, Flanigan A and Chen J: Phosphatidic acid-mediated mitogenic activation of mTOR signaling. Science 294: 1942-1945, 2001.

30. Sengupta S, Peterson TR and Sabatini DM: Regulation of the mTOR complex 1 pathway by nutrients, growth factors, and stress. Mol Cell 40: 310-322, 2010.

31. Finn RS: Current and future treatment strategies for patients with advanced hepatocellular carcinoma: Role of mTOR inhibition. Liver Cancer 1: 247-256, 2012.

32. Pyo JO, Nah J and Jung YK: Molecules and their functions in autophagy. Exp Mol Med 44: 73-80, 2012.

33. Bayascas JR and Alessi DR: Regulation of Akt/PKB Ser473 phosphorylation. Mol Cell 18: 143-145, 2005.

34. Perry JA, Kiezun A, Tonzi P, Van Allen EM, Carter SL, Baca SC, Cowley GS, Bhatt AS, Rheinbay E, Pedamallu CS, et al: Complementary genomic approaches highlight the PI3K/mTOR pathway as a common vulnerability in osteosarcoma. Proc Natl Acad Sci USA 111: E5564-E5573, 2014.

35. Kuijjer ML, van den Akker BE, Hilhorst R, Mommersteeg M, Buddingh EP, Serra M, Bürger $\mathrm{H}$, Hogendoorn $\mathrm{PC}$ and Cleton-Jansen AM: Kinome and mRNA expression profiling of high-grade osteosarcoma cell lines implies Akt signaling as possible target for therapy. BMC Med Genomics 7: 4, 2014.

36. Pópulo H, Lopes JM and Soares P: The mTOR signalling pathway in human cancer. Int J Mol Sci 13: 1886-1918, 2012.

37. Freeman SS, Allen SW, Ganti R, Wu J, Ma J, Su X, Neale G, Dome JS, Daw NC and Khoury JD: Copy number gains in EGFR and copy number losses in PTEN are common events in osteosarcoma tumors. Cancer 113: 1453-1461, 2008.

38. Zhou Q, Deng Z, Zhu Y, Long H, Zhang S and Zhao J: mTOR/p70S6K signal transduction pathway contributes to osteosarcoma progression and patients' prognosis. Med Oncol 27: 1239-1245, 2010.

39. Di Cristofano C, Leopizzi M, Miraglia A, Sardella B, Moretti V, Ferrara A, Petrozza V and Della Rocca C: Phosphorylated ezrin is located in the nucleus of the osteosarcoma cell. Mod Pathol 23: 1012-1020, 2010.

40. Wan X, Mendoza A, Khanna C and Helman LJ: Rapamycin inhibits ezrin-mediated metastatic behavior in a murine model of osteosarcoma. Cancer Res 65: 2406-2411, 2005.

41. Tanaka M, Yamazaki T, Araki N, Yoshikawa H, Yoshida T, Sakakura $\mathrm{T}$ and Uchida A: Clinical significance of tenascin-C expression in osteosarcoma: Tenascin-C promotes distant metastases of osteosarcoma. Int J Mol Med 5: 505-510, 2000.

42. Zheng L, Zhang D, Zhang Y, Wen Y and Wang Y: mTOR signal transduction pathways contribute to TN-C FNIII A1 overexpression by mechanical stress in osteosarcoma cells. Mol Cells 37: 118-125, 2014.

43. Zhao S, Lu N, Chai Y and Yu X: Rapamycin inhibits tumor growth of human osteosarcomas. J BUON 20: 588-594, 2015.

44. Zhang J, Yu XH, Yan YG, Wang C and Wang WJ: PI3K/Akt signaling in osteosarcoma. Clin Chim Acta 444: 182-192, 2015.

45. Goudarzi KM, Nistér M and Lindström MS: mTOR inhibitors blunt the p53 response to nucleolar stress by regulating RPL11 and MDM2 levels. Cancer Biol Ther 15: 1499-1514, 2014.

46. Song R, Tian K, Wang W and Wang L: P53 suppresses cell proliferation, metastasis, and angiogenesis of osteosarcoma through inhibition of the PI3K/AKT/mTOR pathway. Int J Surg 20: 80-87, 2015. 
47. Wang X, Lai P, Zhang Z, Huang M, Wang L, Yin M, Jin D, Zhou $\mathrm{R}$ and Bai X: Targeted inhibition of mTORC2 prevents osteosarcoma cell migration and promotes apoptosis. Oncol Rep 32: 382-388, 2014.

48. Fleuren ED, Versleijen-Jonkers YM, Roeffen MH, Franssen GM, Flucke UE, Houghton PJ, Oyen WJ, Boerman OC and van der Graaf WT: Temsirolimus combined with cisplatin or bevacizumab is active in osteosarcoma models. Int J Cancer 135 2770-2782, 2014.

49. Wagner LM, Fouladi M, Ahmed A, Krailo MD, Weigel B, DuBois SG, Doyle LA, Chen H and Blaney SM: Phase II study of cixutumumab in combination with temsirolimus in pediatric patients and young adults with recurrent or refractory sarcoma: A report from the Children's Oncology Group. Pediatr Blood Cancer 62: 440-444, 2015.

50. Xie ZG, Xie Y and Dong QR: Inhibition of the mammalian target of rapamycin leads to autophagy activation and cell death of MG63 osteosarcoma cells. Oncol Lett 6: 1465-1469, 2013.

51. Horie R, Nakamura O, Yamagami Y, Mori M, Nishimura H, Fukuoka $\mathrm{N}$ and Yamamoto T: Apoptosis and antitumor effects induced by the combination of an mTOR inhibitor and an autophagy inhibitor in human osteosarcoma MG63 cells. Int J Oncol 48: 37-44, 2016.

52. Gupte A, Baker EK, Wan SS, Stewart E, Loh A, Shelat AA, Gould CM, Chalk AM, Taylor S, Lackovic K, et al: Systematic Screening identifies dual PI3K and mTOR inhibition as a conserved therapeutic vulnerability in osteosarcoma. Clin Cancer Res 21: 3216-3229, 2015.

53. Zhu YR, Min H,Fang JF,Zhou F, Deng XW andZhang YQ: Activity of the novel dual phosphatidylinositol 3-kinase/mammalian target of rapamycin inhibitor NVP-BEZ235 against osteosarcoma. Cancer Biol Ther 16: 602-609, 2015.

54. Gobin B, Battaglia S, Lanel R, Chesneau J, Amiaud J, Rédini F, Ory B and Heymann D: NVP-BEZ235, a dual PI3K/mTOR inhibitor, inhibits osteosarcoma cell proliferation and tumor development in vivo with an improved survival rate. Cancer Lett 344: 291-298, 2014.

55. Niu NK, Wang ZL, Pan ST, Ding HQ, Au GH, He ZX, Zhou ZW, Xiao G, Yang YX, Zhang X, et al: Pro-apoptotic and proautophagic effects of the Aurora kinase A inhibitor alisertib (MLN8237) on human osteosarcoma U-2 OS and MG-63 cells through the activation of mitochondria-mediated pathway and inhibition of p38 MAPK/PI3K/Akt/mTOR signaling pathway. Drug Des Devel Ther 9: 1555-1584, 2015.

56. Slotkin EK, Patwardhan PP, Vasudeva SD, de Stanchina E, Tap WD and Schwartz GK: MLN0128, an ATP-competitive mTOR kinase inhibitor with potent in vitro and in vivo antitumor activity, as potential therapy for bone and soft-tissue sarcoma. Mol Cancer Ther 14: 395-406, 2015.
57. Grignani G, Palmerini E, Ferraresi V, D'Ambrosio L, Bertulli R, Asaftei SD, Tamburini A, Pignochino Y, Sangiolo D, Marchesi E, et al; Italian Sarcoma Group: Sorafenib and everolimus for patients with unresectable high-grade osteosarcoma progressing after standard treatment: A non-randomised phase 2 clinical trial. Lancet Oncol 16: 98-107, 2015.

58. Chawla SP, Staddon AP, Baker LH, Schuetze SM, Tolcher AW, D'Amato GZ, Blay JY, Mita MM, Sankhala KK, Berk L, et al: Phase II study of the mammalian target of rapamycin inhibitor ridaforolimus in patients with advanced bone and soft tissue sarcomas. J Clin Oncol 30: 78-84, 2012.

59. Liu Y, Bi T, Dai W, Wang G, Qian L, Shen G and Gao Q: Lupeol induces apoptosis and cell cycle arrest of human osteosarcoma cells through PI3K/AKT/mTOR pathway. Technol Cancer Res Treat: Oct 6, 2015 (Epub ahead of print). pii: 1533034615609014

60. Liu WN, Lin JH, Cheng YR, Zhang L, Huang J, Wu ZY, Wang FS, Xu SG, Lin WP, Lan WB, et al: FIM-A, a phosphoruscontaining sirolimus, inhibits the angiogenesis and proliferation of osteosarcomas. Oncol Res 20: 319-326, 2013.

61. Zhou R, Zhang Z, Zhao L, Jia C, Xu S, Mai Q, Lu M, Huang M, Wang L, Wang X, et al: Inhibition of mTOR signaling by oleanolic acid contributes to its anti-tumor activity in osteosarcoma cells. J Orthop Res 29: 846-852, 2011.

62. Li G, Cai M, Fu D, Chen K, Sun M, Cai Z and Cheng B: Heat shock protein $90 \mathrm{~B} 1$ plays an oncogenic role and is a target of microRNA-223 in human osteosarcoma. Cell Physiol Biochem 30: 1481-1490, 2012.

63. Lin S, Shao NN, Fan L, Ma XC, Pu FF and Shao ZW: Effect of microRNA-101 on proliferation and apoptosis of human osteosarcoma cells by targeting mTOR. J Huazhong Univ Sci Technolog Med Sci 34: 889-895, 2014.

64. Yao C, Wei JJ, Wang ZY, Ding HM, Li D, Yan SC, Yang YJ and $\mathrm{Gu} \mathrm{ZP}$ : Perifosine induces cell apoptosis in human osteosarcoma cells: New implication for osteosarcoma therapy? Cell Biochem Biophys 65: 217-227, 2013.

65. Moriceau G, Ory B, Mitrofan L, Riganti C, Blanchard F, Brion R, Charrier C, Battaglia S, Pilet P, Denis MG, et al: Zoledronic acid potentiates mTOR inhibition and abolishes the resistance of osteosarcoma cells to RAD001 (Everolimus): Pivotal role of the prenylation process. Cancer Res 70: 10329-10339, 2010.

66. Jiang $\mathrm{H}$ and Zeng Z: Dual mTORC1/2 inhibition by INK-128 results in antitumor activity in preclinical models of osteosarcoma. Biochem Biophys Res Commun 468: 255-261, 2015. 